\title{
Trends in stroke mortality in Greater London and south east England-evidence for a cohort effect?
}

\author{
Ravi Maheswaran, David P Strachan, Paul Elliott, Martin J Shipley
}

\begin{abstract}
Objective and setting - To examine time trends in stroke mortality in Greater London compared with the surrounding South East Region of England.

Design - Age-cohort analysis based on routine mortality data.

Subjects - Resident population aged 45 years or more.

Main outcome measure - Age specific stroke mortality rates, 1951-92.

Main results - In 1951, stroke mortality was lower in Greater London than the surrounding South East Region in all age bands over 45 . It has been declining in both areas but the rate of decline has been significantly slower in Greater London $(p<0.0001)$. The differences in rates of decline were such that stroke mortality is now higher in Greater London for people under 75. The crossover of age specific stroke mortality rates occurred at different periods in different age bands and is consistent with a cohort effect, with similar rates in Greater London and the surrounding south east for men and women born around 1916-21. This cohort effect does not appear to be consistent with past maternal and neonatal mortality rates in these areas, nor, within the limitations of the data, with the ethnic composition of cohorts.

Conclusions - There seems to be a cohort effect on stroke mortality which is not explained by past maternal and neonatal mortality. If the decline in stroke mortality continues at its current rate, the Health of the Nation stroke target is unlikely to be achieved in Greater London.
\end{abstract}

(f Epidemiol Community Health 1997;51:121-126)

Department of Epidemiology and Public Health, Imperial College School of Medicine at St Mary's, London

P Elliott

Department of Epidemiology and Public Health, University College London Medical School, London M J Shipley

Correspondence to: Dr R Maheswaran Department of Epidemiology and Public Health, Imperial College School of Medicine London W2 1 PG.

Accepted for publication June 1996 to London to work in the domestic services where they generally had a nutritious diet. A study of proportional mortality from stroke suggested that persons born in London retained their lower risk of stroke when they moved elsewhere, ${ }^{3}$ but another study which used standardised mortality ratios suggested the opposite - people who moved to London acquired a lower risk of fatal stroke. ${ }^{4}$

While standardised mortality ratios for stroke for all ages in Greater London are low, Health of the Nation indicators for district health authorities suggest that stroke mortality for Greater London relative to other areas may vary with age. ${ }^{5}$

The aim of this study was to examine time trends for stroke mortality in Greater London compared with the surrounding South East Region in relation to age, period of death, and birth cohort.

\section{Methods}

Routine data on stroke deaths (International Classification of Disease 8th and 9th revisions 430-438; 6th and 7th revisions 330-334) and mid-year population estimates were obtained from the Registrar General's Statistical Reviews of England and Wales for the period 1951-1973 and from the Office of Population Censuses and Surveys annual Area Mortality series from 1974-92. ${ }^{16}$

The South East Region is currently one of eight standard regions in England based on the Registrar General's classification. Greater London and the South East Region were defined in 1931. Since then, the main boundary changes have been in 1950, 1965, and 1974 . However, these did not noticeably affect trends in stroke mortality rates.

Maternal (1901-30) and neonatal (1911-30) mortality were used as proxy measures of the early-life environment. ${ }^{6}$ There were no data on neonatal deaths before 1911. The period in which these proxy measures were examined was before Greater London and the surrounding south east had been defined. Data on existing areas were therefore amalgamated to correspond to these two areas.

The analysis required an indication of the ethnic composition of birth cohorts. However, population ethnicity data were only available from the 1991 census, the first census in England and Wales to collect these data. ${ }^{7}$ These data were used to estimate the ethnic composition of cohorts (eg, the Afro-Caribbean population in Greater London in the 50-54 

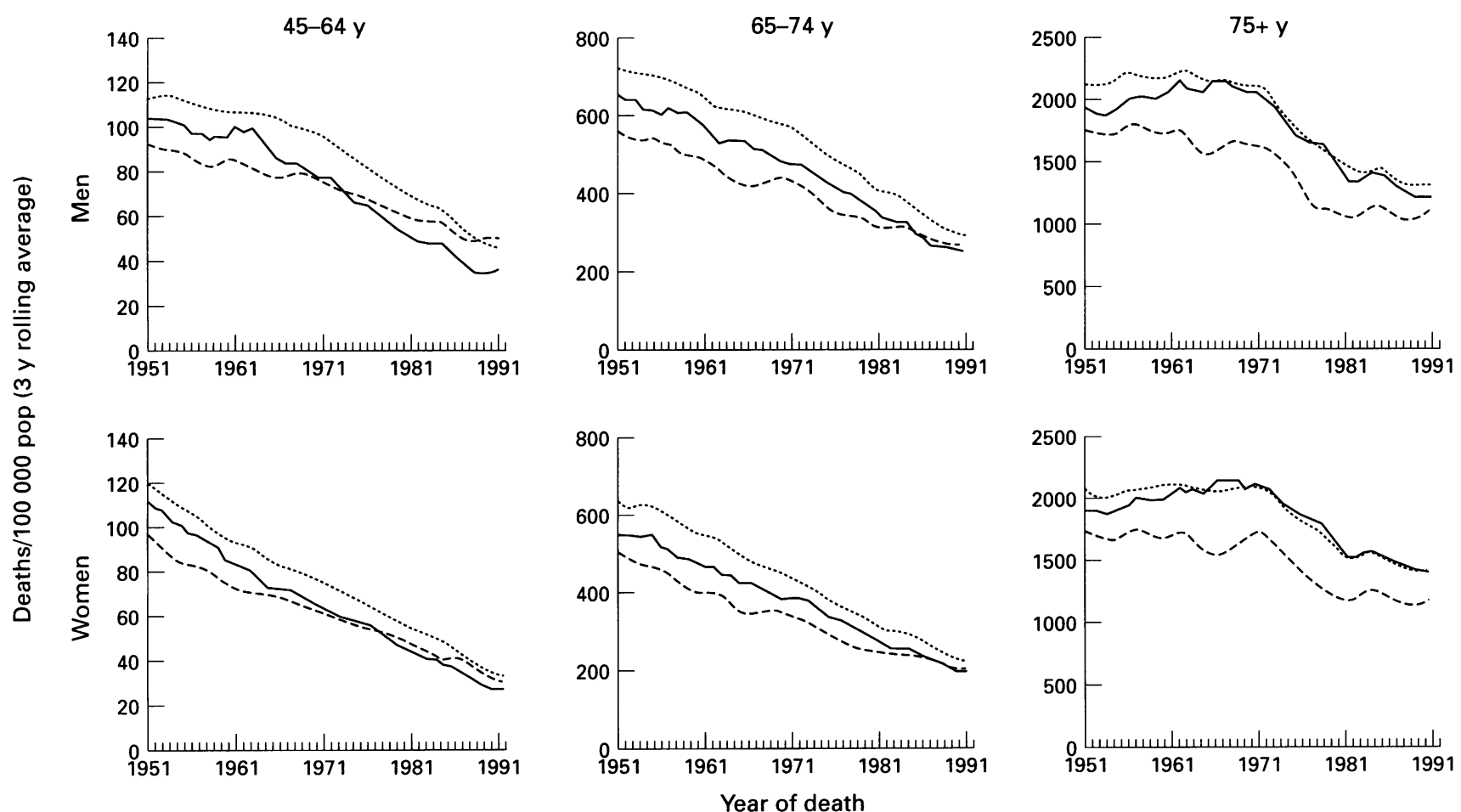

Figure 1 Trends in stroke mortality for Greater London, the South East Region, and England and Wales between 1951 and 1992 inclusive.

age band was $8.3 \%$ and this was taken to represent the percentage of Afro-Caribbeans in the 1937 to 1941 birth cohorts. This does not, however, make any assumptions about place of birth). The census categories were amalgamated into three groups: Afro-Caribbeans, Asians and others, and Irish born migrants. The limitations of this method are addressed in the Discussion.

\section{STATISTICAL ANALYSIS}

The patterns in stroke mortality rates for Greater London and the surrounding south east were examined graphically, using three year rolling averages (with two year averages at the start and end of the time period examined) to reduce the effect of random variation. All rates for the surrounding south east were calculated having excluded Greater London.

Log linear Poisson regression analysis was used to assess if the rate of decline in stroke mortality in Greater London in relation to birth cohort was significantly different from that in the surrounding south east and to see if ethnicity could account for this difference. ${ }^{8}$ An age cohort model was fitted. ${ }^{910}$ Age and area were entered as categorical variables while cohort (ie, year of birth) was entered as a continuous variable. An area-by-cohort interaction term, which represented the difference in rates of decline between the two areas, was included. The percentages of ethnic minorities in each cohort were then entered as continuous variables to see if they reduced the magnitude of this area-by-cohort interaction term. Data on men and women were analysed separately. Statistical analysis was carried out using GLIM. ${ }^{11}$ Some residual variation in excess of the Poisson distribution (overdispersion) was observed. Adjustment for this overdispersion made little difference to the degree of significance of statistical tests.

\section{Results}

TRENDS IN STROKE MORTALITY

Trends in mortality from all cerebrovascular diseases from 1951-92 in three age bands for men and women are shown in figure 1. The overall pattern was similar for men and women.

Stroke mortality was lower in Greater London than the surrounding south east in all three age groups in 1951. The rate of subsequent decline was slower in Greater London than in the surrounding south east, where the age specific rates of decline were closer to those in England and Wales as a whole. Stroke mortality rates in Greater London and the surrounding south east crossed over around 1973 in the 45-64 year age group and around 1986 in the 65-74 year age group. No crossover has yet occurred in the $75+$ age group. 


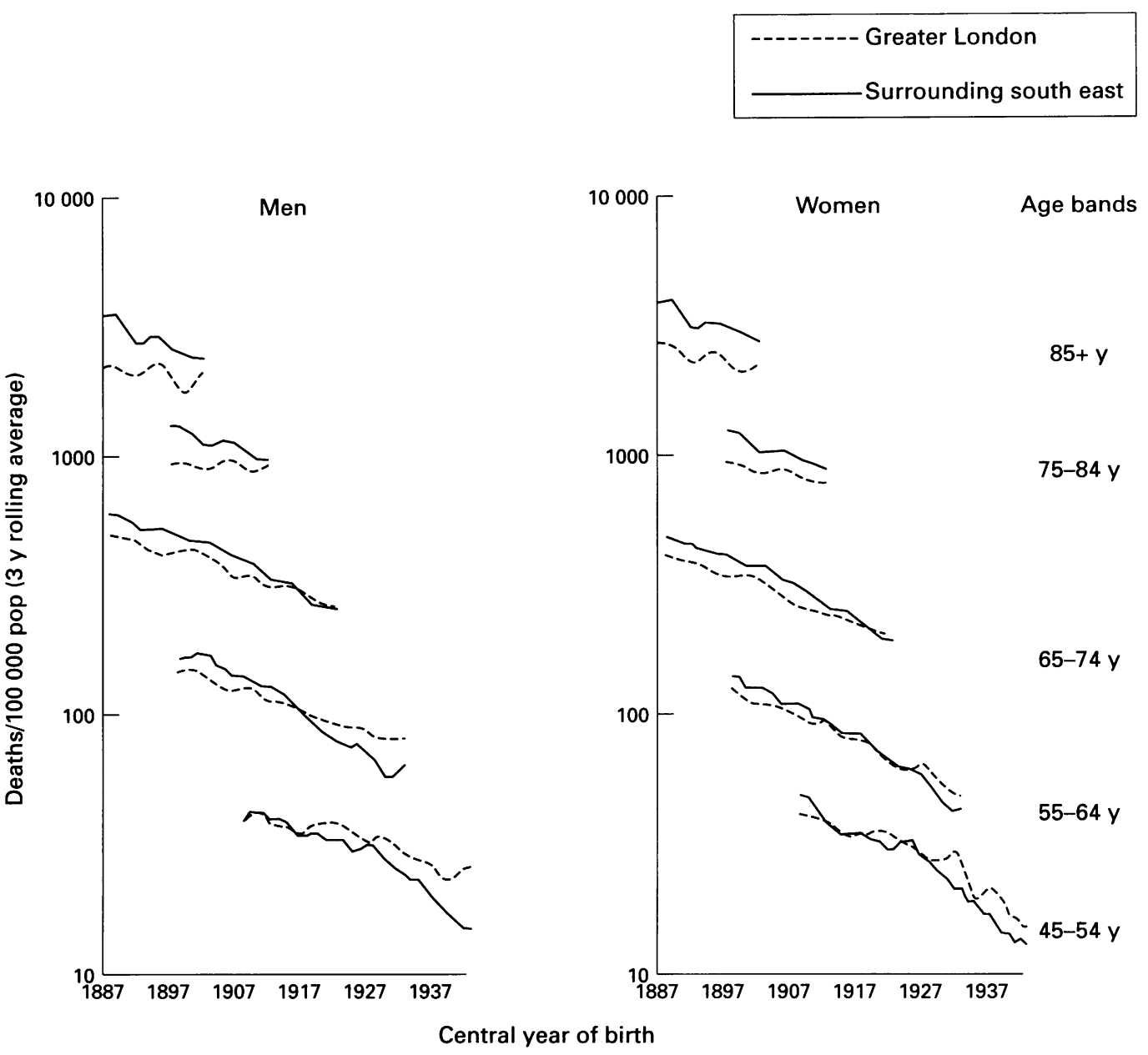

Figure 2 Age specific stroke mortality rates in relation to year of birth in Greater London and the surrounding South East Region, 1887-1942.

\section{STROKE MORTALITY IN RELATION TO YEAR OF} BIRTH

It is usually not possible to attribute linear drifts in a set of age specific mortality rates to either a period (ie, year of death) effect or a cohort (ie, year of birth) effect unless there are kinks (eg, changes in direction) in these rates. If there are kinks, rates may be plotted in relation to year of birth and year of death. If the kinks all occur around the same year when plotted by year of birth, the effect is consistent with a cohort effect. Similarly, if the kinks all occur around a particular year when plotted by year of death, the effect is consistent with a period effect. In this study, although there were no kinks in the rates, there were two sets of rates which crossed over and the crossover points are the equivalent of kinks.

The crossover points occurred sequentially when plotted by year of death and the pattern is not consistent with a period effect (fig 1). In order to see if the crossover pattern is consistent with a cohort effect, rates were plotted by year of birth. Five narrower age bands were used in order to examine this effect more clearly. Published data in these age bands were available for shorter time spans.

For both men and women, stroke mortality in Greater London was lower than that in the surrounding south east in all five age bands in the earlier birth cohorts (fig 2). However, the rate of decline was significantly slower in Greater London than the surrounding south east $(p<0.0001)$. This slower rate of decline was seen in all five age bands. The differential rate of decline resulted in a crossover of rates in the three younger age bands, giving a pattern consistent with a cohort effect. The crossover point was estimated from the regression models to be in the birth year of 1916 for men and 1921 for women.

This cohort effect is illustrated by figure 3 , which shows the ratio (expressed as a percentage) of stroke mortality rates in Greater London relative to the surrounding south east in relation to age band for birth cohorts from 1887-1942. Among older cohorts, the ratio was less than $100 \%$, indicating that stroke mortality was lower in Greater London than the surrounding south east. The magnitude of this ratio increased with successive cohorts and between 1910 and 1920 the rates for cohorts in both areas were similar. In subsequent cohorts the ratio tended to be greater than $100 \%$, with a progressive increase, indicating that rates for these cohorts were now higher, and increasingly so, in Greater London compared with the surrounding south east. 


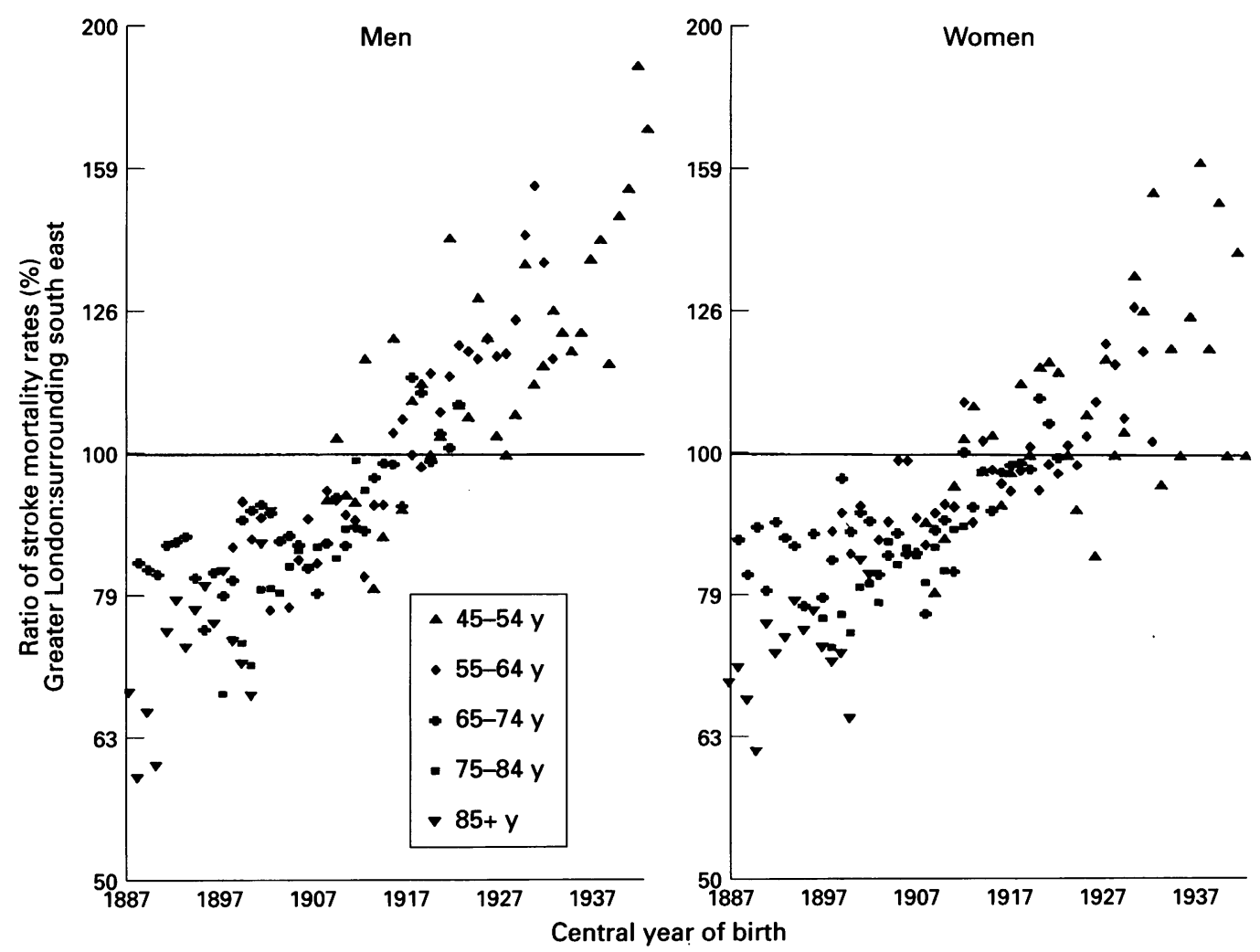

Figure 3 Ratio (\%) of age specific stroke mortality rates (Greater London: surrounding South East Region) in relation to year of birth, 1887-1942.

\section{POSSIBLE EXPLANATIONS FOR THE COHORT} EFFECT

If the differential rates of decline in recent stroke mortality rates were attributable to differences in the intrauterine environment in the two areas earlier this century, ${ }^{2}$ then the pattern one might expect to see for maternal and neonatal mortality (proxy measures of the intrauterine environment) would be declining rates in both Greater London and the surrounding south east, with lower rates in Greater London before 1916. Greater London would, however, be expected to have had a slower rate

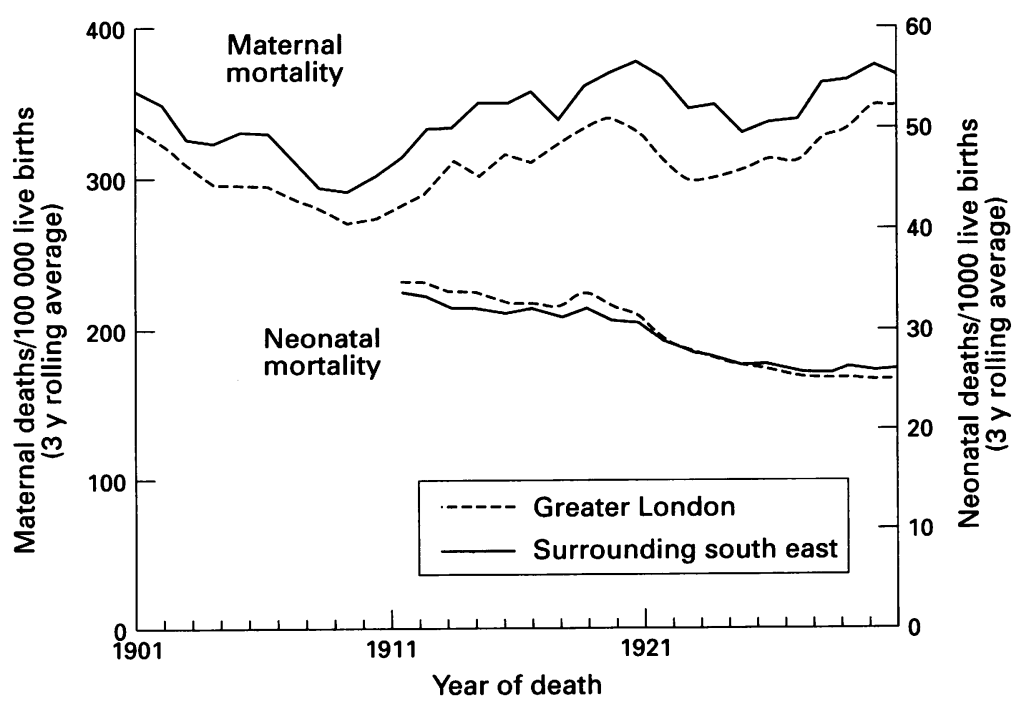

Figure 4 Maternal and neonatal mortality in Greater London and the surrounding South East Region, 1901-30. of decline. A crossover around 1916-21 might be expected with subsequent rates being higher in Greater London than the surrounding south east, mirroring the pattern seen for stroke mortality.

However, maternal mortality from all causes was lower in Greater London than the surrounding south east throughout the period 1901-30 with no evidence of a decline in either area (fig 4). Maternal mortality excluding deaths from puerperal sepsis also showed a similar pattern (not shown).

Neonatal mortality declined sharply but was similar in both areas (fig 4). A crossover occurred but in the opposite direction to that expected, starting slightly higher in Greater London in 1911 and being slightly lower by 1930 compared with the surrounding south east.

The recent progressive increase in the ethnic minority composition of population cohorts in Greater London compared with the surrounding south east was examined as another possible explanation for the slower rate of decline in stroke mortality in Greater London, as some ethnic groups, particularly Afro-Caribbeans, are known to have high risks of death from stroke. ${ }^{12}$ However, the rate of decline in stroke mortality for Greater London cohorts was slower than that for the surrounding south east well before any appreciable increase in the percentage of Afro-Caribbeans or of other ethnic groups (table 1). Statistically, inclusion of the ethnic minority groups in the regression models did not reduce the magnitude of the area-by-cohort interaction term. When the stat- 
Table 1 Percentages of ethnic minorities in Greater London and the surrounding South East Region in relation to birth cohort, estimated from the 1991 census

\begin{tabular}{|c|c|c|c|c|c|c|}
\hline \multirow[b]{2}{*}{ Cohort } & \multicolumn{3}{|l|}{ Greater London } & \multicolumn{3}{|c|}{ Surrounding South East Region } \\
\hline & Afro-Caribbeans & Asians and others & Irish born & Afro-Caribbeans & Asians and others & Irish born \\
\hline $\begin{array}{l}\text { Men: } \\
1902 \\
1907 \\
1912 \\
1917 \\
1922 \\
1927 \\
1932 \\
1937 \\
1942\end{array}$ & $\begin{array}{l}0.9 \\
0.9 \\
1.4 \\
2.5 \\
4.0 \\
6.2 \\
7.7 \\
8.3 \\
5.3\end{array}$ & $\begin{array}{r}2.3 \\
2.0 \\
2.5 \\
3.8 \\
4.9 \\
7.5 \\
10.6 \\
12.7 \\
10.7\end{array}$ & $\begin{array}{l}2.3 \\
3.0 \\
4.5 \\
5.3 \\
5.7 \\
6.3 \\
6.8 \\
6.7 \\
6.4\end{array}$ & $\begin{array}{l}0.1 \\
0.1 \\
0.1 \\
0.2 \\
0.3 \\
0.4 \\
0.7 \\
0.7 \\
0.5\end{array}$ & $\begin{array}{l}0.4 \\
0.3 \\
0.3 \\
0.5 \\
0.8 \\
1.3 \\
1.9 \\
2.4 \\
2.0\end{array}$ & $\begin{array}{l}1.2 \\
1.4 \\
1.8 \\
2.2 \\
2.2 \\
2.3 \\
2.5 \\
2.5 \\
2.3\end{array}$ \\
\hline $\begin{array}{l}\text { Women: } \\
1902 \\
1907 \\
1912 \\
1917 \\
1922 \\
1927 \\
1932 \\
1937 \\
1942\end{array}$ & $\begin{array}{l}0.6 \\
0.6 \\
1.0 \\
1.7 \\
2.7 \\
4.9 \\
7.0 \\
8.1 \\
7.1\end{array}$ & $\begin{array}{r}1.1 \\
1.4 \\
1.9 \\
2.9 \\
3.8 \\
5.6 \\
7.9 \\
10.4 \\
10.9\end{array}$ & $\begin{array}{l}2.3 \\
3.1 \\
4.5 \\
5.5 \\
5.9 \\
6.7 \\
7.3 \\
7.0 \\
6.6\end{array}$ & $\begin{array}{l}0.1 \\
0.1 \\
0.1 \\
0.1 \\
0.2 \\
0.3 \\
0.5 \\
0.7 \\
0.6\end{array}$ & $\begin{array}{l}0.2 \\
0.2 \\
0.3 \\
0.5 \\
0.6 \\
0.9 \\
1.3 \\
1.8 \\
1.9\end{array}$ & $\begin{array}{l}1.2 \\
1.6 \\
2.1 \\
2.6 \\
2.5 \\
2.6 \\
2.7 \\
2.7 \\
2.5\end{array}$ \\
\hline
\end{tabular}

The term "birth cohort" does not imply that ethnic groups were born in these two areas.

istical analysis was restricted to mortality data from 1982 onwards, limiting the extent of retrospective estimation of the ethnic minority cohort composition, the results were similar.

\section{Discussion}

The results suggest that factors exerting a cohort effect on stroke mortality might explain the initial lower stroke mortality and the slower rate of decline in Greater London compared with the surrounding South East Region of England.

A cohort related change in the ratio of stroke mortality in Greater London to the surrounding south east would be consistent with changing patterns of exposure in early life but our analysis offers no support for the more specific hypothesis that intrauterine development, as indicated by past maternal and neonatal mortality rates, is the influential factor. ${ }^{2}$ However, we make the assumption that most of those who died of stroke in Greater London and the surrounding south east were also born in the same two areas. Population movements, particularly around the second world war, could have masked any underlying association.

The ethnic minority composition of cohorts showed differing progressive increases in the two areas but this did not appear to be a major explanatory factor for the cohort effect. This observation has to be interpreted with caution due to limitations of estimating the ethnic composition of cohorts from a single census, eg recent immigration could have led to an overestimate of the ethnic minority composition of older cohorts in past decades. However, when the analysis was limited to data from 1982 onwards, by which time the main episodes of migration from Commonwealth countries had occurred, similar results were obtained.

Factors which could be classified as exerting a period effect on stroke mortality - including anti-hypertensive treatment, reduction in sodium intake, and improved survival following stroke - might account for part of the reduction in stroke mortality in recent years but would be unlikely sources of the cohort related pattern that we observed. ${ }^{13-17}$

A number of biases may have affected the comparison of stroke mortality rates in Greater London and the surrounding South East Region. The population in Greater London may have been slightly younger even within 10 year age bands; wealthy elderly people may have migrated from the capital to the surrounding south east; frail elderly people may have moved to nursing homes in the surrounding south east; or there may have been variations in diagnosis and medical care of cerebrovascular disease or in certification of stroke as a cause of death. However, it seems unlikely that any of these could have artefactually generated a cohort related change in the ratio of stroke mortality rates in the two areas.

There is an apparent discrepancy between standardised mortality ratios for stroke, which were lower for Greater London than for the surrounding south east, and age specific mortality rates, which are now higher in Greater London in the younger age bands. This is because standardised mortality ratios largely reflect the pattern in the older age bands among whom the majority of stroke deaths occur and for whom stroke mortality is still lower in Greater London.

The Health of the Nation target is a minimum $40 \%$ reduction in stroke mortality from the baseline rate in $1990 .{ }^{18}$ From the regression models, the predicted reductions in stroke mortality between 1990 and 2000 for Greater London are $16 \%$ for men and $18 \%$ for women. Predictions need to be treated with caution. Nevertheless, the data suggest that if the decline in stroke mortality in Greater London continues at its current rate, the stroke target is unlikely to be achieved. Consideration therefore needs to be given to enhancing public health measures which could reduce stroke mortality. Socioeconomic deprivation and ethnicity have been shown to be important in explaining geographical variability, suggesting that preventative action is overdue. ${ }^{19}$ 
1 Office of Population Censuses and Surveys. Area mortality. London: HMSO, 1974-92.

2 Barker DJP, Osmond C, Pannett B. Why Londoners have low death rates from ischaemic heart disease and stroke. BMf 1992;305:1551-4.

3 Osmond C, Slattery JM, Barker DJP. Mortality by place of birth. In: Britton M, ed. Office of Population Censuses and birth. In: Britton M, ed. Office of Population Censuses and
Surveys. Mortality and geography - the Registrar General's Surveys. Mortality and geography - the Registrar General
decennial supplement for England and Wales (1979-1983) decennial supplement for England

4 Strachan DP, Leon DA, Dodgeon B. Mortality from cardiovascular disease among interregional migrants in England and Wales. BMF 1995;310:423-7.

5 Department of Health. Common data set - health of the nation baseline indicators. London: Department of Health, 1992.

6 Registrar General's statistical reviews of England and Wales. Part 1: Tables, Medical. London: HMSO, 1901-1973.

7 Office of Population Censuses and Surveys. 1991 Census. London: HMSO, 1993.

8 Breslow NE, Day NE. Statistical methods in cancer research Vol II - the design and analysis of cohort studies. Lyon: IARC, 1977.

9 Clayton D, Schifflers E. Models for temporal variation in cancer rates. I: Age-period and age-cohort models. Stat Med 1987;6:449-67.

10 Clayton D, Schifflers E. Models for temporal variation in cancer rates. II: Age-period-cohort models. Stat Med 1987; 6:469-81.

11 Baker RJ, Nelder JA. The GLIM System, Release 3. Oxford: Numerical Algorithms Group, 1978.

12 Balarajan R. Ethnic differences in mortality from ischaemic Balarajan R. Ethnic differences in mortality from ischaemic
heart disease and cerebrovascular disease in England and Wales. BMf 1991;302:560-4

13 McGovern PG, Burke GL, Sprafka JM, Xue S, Folsom AR, Blackburn $H$. Trends in mortality, morbidity, and risk factor levels for stroke from 1960 through 1990 - The Minnesota heart survey. $\mathcal{F} A M A$ 1992;268:753-9.

14 Kesteloot H, Sasaki S, Xie J, Joossens JV. Secular trends in cerebrovascular mortality. F Hum Hypertens 1994;8:401-7.

15 Stamler J, Rose G, Stamler R, Elliott P, Dyer A, Marmo M. INTERSALT study findings - Public health and medical care implications. Hypertension 1989;14:570-7.

16 Bonita R, Beaglehole R. Explaining stroke mortality trends. Lancet 1993;341:1510-1.

17 Shahar E, McGovern PG, Sprafka JM, et al. Improved survival of stroke patients during the 1980s - The Minnesota stroke survey. Stroke 1995;26:1-6.

18 Department of Health. The health of the nation. London: HMSO, 1992.

19 Maheswaran R, Elliott P, Strachan DP. Socioeconomic deprivation, ethnicity and stroke mortality in Greater London and south east England. $\mathcal{F}$ Epidemiol Community Health 1997;51:127-31. 